\title{
IMAGE SETS OF FOLDING SURFACES
}

ANA M. D’AZEVEDO BREDA

Received 27 January 2004

Isometric foldings are a special class of length-preserving maps of Riemannian manifolds and were initially studied by S. Robertson. For an explanation of their topological and combinatorial properties, see the related works of Ana Breda, Altino Santos, M. El-Ghoul, and E. M. Elkholy. Here, we explore some properties of the singular set and describe the image set of planar, spherical, and hyperbolic foldings.

\section{Introduction}

The notion of isometric foldings of Riemannian manifolds was introduced in 1977 by Robertson [2].

An isometric folding $f: M \rightarrow N$ is a map which sends piecewise geodesic segments in piecewise geodesic segments of the same length. An isometric folding is a continuous local isometry, which need not be differentiable. The points where it fails to be differentiable are called singular points.

We will denote by $\mathscr{I}(M, N)$ the set of isometric foldings from $M$ to $N$.

In [2], the structure of the singular set of an isometric folding was given. For the particular case of isometric foldings of surfaces, $f: S_{1} \rightarrow S_{2}$, it was shown that there is a decomposition of $S_{1}$ into mutually disjoint, connected totally geodesic submanifolds, called strata, such that

(i) $S_{f}=S_{f}^{0} \cup S_{f}^{1}$ where $S_{f}^{i}$ denotes the union of all strata of dimension $i, i=0,1$;

(ii) the restriction of $f$ to each stratum is a locally isometric immersion into $S_{2}$;

(iii) the frontier of each stratum is a stratum of lower dimension, and in case $S_{1}$ is compact of finitely many such strata;

(iv) the frontier of $S_{f}^{i}, i=1,2$, in $S_{1}$ is the union of all sets $S_{f}^{j}, j=0, i-1$;

(v) $S_{f}$ is either the empty set or it can be regarded as a graph of even valency satisfying the angle folding relation, that is, the sums of alternating angles are $\pi$. 

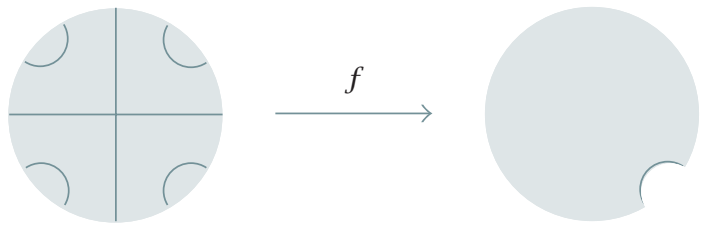

Figure 2.1. Singular set of a hyperbolic folding.

\section{Finite foldings of constant curvature surfaces}

In this section, we will focus our attention on isometric foldings $f: S \rightarrow S$ of surfaces of constant curvature whose set of singularities, $S_{f}$, partitions $S$ into a finite number of regions (faces). Such maps will be called finite foldings.

Let $S$ be a surface of constant curvature. We will denote by $\mathscr{F}(S)$ the set of all finite isometric foldings of $S$.

Given a finite folding $f: S \rightarrow S$ with a nonempty set of singularities, the connected components of $S_{f}^{2}, S_{f}^{1}$, and $S_{f}^{0}$ will be called, respectively, faces, edges, and vertices.

Proposition 2.1. If $f \in \mathscr{F}(S)$, then there are no strata of dimension 0 if there are no strata of dimension 1.

Proof. If there are no strata of dimension 1, then the stratification determined on $S$ by $f$ forces $S$ to have only one face, say $F$.

Assume now that strata of dimension 0 are also present. Let $x$ be a point on $F, v$ a vertex on $S$, and $\gamma: \mathbb{R} \rightarrow S$ a geodesic segment such that $\gamma(0)=x, \gamma(t)=v,\left(t \in \mathbb{R}^{+}\right)$, and $\int_{0}^{t}\|\gamma(t)\|=d_{S}(x, v)$, inducing a piecewise geodesic segment $\gamma_{\star}=f \circ \gamma$ on $S$, for which $t$ is the smallest positive singularity of $\gamma_{\star}$. Choose $\delta>0$ sufficiently small in order to have $t$ as the unique singularity of $\gamma_{\star}$ on $[0, t+\delta]$.

Now, consider the point $y=\gamma(t+\varepsilon) t<\varepsilon<t+\delta$. On one hand, $d_{S}(f(x), f(y))<$ $d_{S}(x, y)$ (the geodesic segment joining $x$ and $y$ contains a singularity of $f$ ) and on the other hand, $d_{S}(f(x), f(y))=d_{S}(x, y)$, since $x, y \in F$ and $f_{\mid F}$ is an isometric immersion, leading to a contradiction.

The same reasoning yields that any $i$-stratum, $i=0,1$, belongs to the frontier of at least two $(i+1)$-strata.

The converse of Proposition 2.1 is false. In fact, the finite folding of the Euclidean plane given by $f(x, y)=(|x|, y)$ determines a stratification of $\mathbb{R}^{2}$ composed by two faces $F_{\zeta}=\left\{(x, y) \in \mathbb{R}^{2}: x_{>} 0\right\}$, one edge $E=\left\{(x, y) \in \mathbb{R}^{2}: x=0\right\}$, and no vertices.

Having in consideration that vertices of the singularity set of an isometric folding obey the angle folding relation, we may deduce the following.

Proposition 2.2. If $f$ is a finite, planar, or spherical folding and $S_{f}^{0}$ is nonempty then $S_{f}$ is connected.

This proposition is not true when $S$ is the hyperbolic plane. It is enough to consider the four foldings illustrated in Figure 2.1. 
Proposition 2.3. Under composition of maps, the set $\mathscr{F}_{(}(S)$ is a semigroup with identity, containing the isometry group of $S$ as a subsemigroup.

Proof. Let $f, g \in \mathscr{F}(S)$. Assume that $A_{1}, A_{2}, \ldots, A_{s}$ and $B_{1}, B_{2}, \ldots, B_{r}$ are the faces determined on $S$ by $f$ and $g$, respectively. Then, $x \in S_{g \circ f}^{2}$ if and only if $x \in A_{i} \cap f^{-1}\left(B_{j}\right)$ for some $i \in\{1, \ldots, s\}$ and some $j \in\{1, \ldots, r\}$.

Since $A_{k} \cap A_{m}=\varnothing$ whenever $k \neq m$ and for every $i=1, \ldots, s, f_{i}=f_{\mid A i}$ is an isometric immersion of $A_{i}$ into $S$, one has

$$
x \in S_{g \circ f}^{2} \Longleftrightarrow x \in A_{i} \cap f_{i}^{-1}\left(B_{j} \cap f_{i}\left(A_{i}\right)\right), \quad i=1, \ldots, s ; j=1, \ldots, r,
$$

and so the number of faces determined by $g \circ f$ is less than or equal to $r s$. The result now follows.

Next, we will show that $\mathscr{F}(S)$ is a subset of $\mathscr{P}(S)$, the set of all continuous proper maps of $S$ (maps for which the inverse images of noncompact sets are not compact sets).

Proposition 2.4. $\mathscr{F}(S) \subset \mathscr{P}(S)$.

Proof. Let $f \in \mathscr{F}_{(}(S)$ and $F_{1}, F_{2}, \ldots, F_{n} n \geq 1$ be the faces determined by $f$. Then, for all $k=1, \ldots, n, f_{\mid F_{k}}$ is an isometric immersion into $S$, and due to the continuity of $f$ so is $f_{\mid \overline{F_{k}}}$. Since $S$ is a complete Hausdorff space, $f\left(\overline{F_{k}}\right)$ is a closed subset of $S$.

Let $K$ be a compact set of $S$. Since $f^{-1}(K)=\bigcup_{k=1}^{n} f_{\mid \overline{F_{k}}}^{-1}\left(f\left(\overline{F_{k}}\right) \cap K\right), f$ is a proper map.

In view of Proposition 2.4, the Hopf degree of a finite folding, denoted by deg, is well defined. See [1] for a comprehensive discourse on the Hopf degree of a map.

Let $f \in \mathscr{F}(S)$. Denoting, respectively, by $V, V^{+}, V^{-}$, and $V_{f}$ the surface area of $S$, of the subset of $S$ where $f$ is an orientation-preserving isometry, of the subset of $S$ where $f$ is an orientation-reversing isometry, and of $f(S)$, it follows immediately that

(i) if $S$ is compact and $\operatorname{deg}(f)=0$, then $V^{+}=V^{-}=(1 / 2) V \geq V_{f}$;

(ii) if $f$ is not surjective, then $\operatorname{deg}(f)=0$;

(iii) if $S$ is compact, then $\operatorname{deg}(f)=0$ or $\operatorname{deg}(f)= \pm 1$ according as $S_{f} \neq \varnothing$ or $S_{f}=\varnothing$.

Let $\mathbb{R}^{2}$ be the Euclidean plane, $S^{2}=\left\{x \in \mathbb{R}^{3}:\|x\|=1\right\}$ the two-sphere, and $\mathbb{H}^{2}=\{x \in$ $\left.\mathbb{R}^{2}:\|x\|<1\right\}$ the hyperbolic plane.

Proposition 2.5. If $f \in \mathscr{F}(S)$ where $S$ is either the Euclidean plane, the two-sphere, or the hyperbolic plane, then

$$
\operatorname{deg}(f)= \begin{cases}0,1,-1 & \text { if } S=\mathbb{R}^{2} \text { or } S=S^{2}, \\ n, n \in \mathbb{Z} & \text { if } S=\mathbb{M}^{2} .\end{cases}
$$

Proof. If $S=S^{2}$, the result follows directly from compactness.

Assume now that $S=R^{2}$. Let $f \in \mathscr{F}\left(R^{2}\right)$. In view of Proposition 2.4, $f$ is a proper map and so the map $g: S^{2} \rightarrow S^{2}$ given by

$$
g(x)= \begin{cases}P_{N} \circ f \circ P_{N}^{-1}(x) & \text { if } x \in S^{2} \backslash N, \\ N & \text { if } x=N,\end{cases}
$$




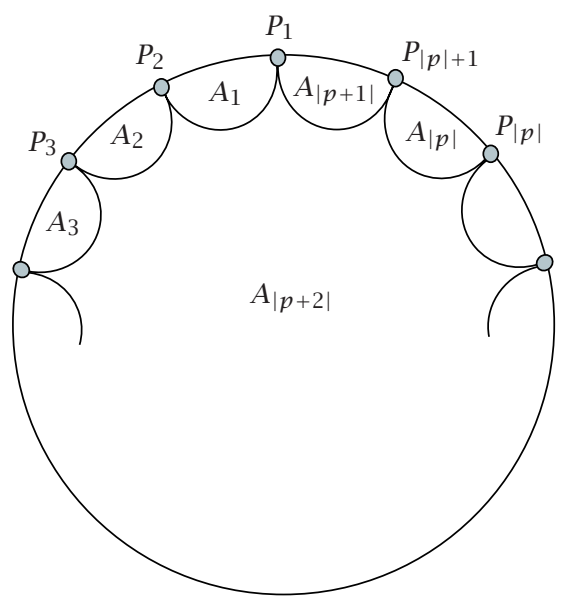

Figure 2.2

where $N=(0,0,1)$ and $P_{N}$ denotes the stereographic projection from the North pole, $N$, is a continuous map. Since $f$ is a finite folding for a sufficiently small neighborhood $U$ of $N$, one has $g^{-1}(U) \subset U$, and the singular set of $g$ in $U$ is either empty or a cone of apex $N$ with an even number of circles emanating from it. Accordingly, the degree of $g$ is either 0 or \pm 1 and so is the degree of $f$.

Suppose now that $S=\mathbb{H}^{2}$. The identity maps, the map $m$ given by $m(x+i y)=-x+i y$ and the map $n$ given by $n(x+i y)=|x|+i y$, are examples of finite hyperbolic foldings of degrees $1,-1$, and 0 , respectively. Let $p \in \mathbb{Z},|p|>1$. Choose $|p|+1$ points, $P_{j}$, correspondent to a subdivision of $S^{1}$, border of $\mathbb{U}^{2}$, into $|p|+1$ equal arcs. The hyperbolic geodesic segment lines $h_{j}$ with end points $P_{j}$ and $P_{j+1}$ partition $\mathbb{U}^{2}$ into $|p|+2$ regions, $A_{j}, j=1, \ldots,|p|+2$, as illustrated in Figure 2.2.

The map $h: \mathbb{W}^{2} \rightarrow \mathbb{M}^{2}$ given by

$$
h(z)= \begin{cases}z & \text { if } z \in A_{|p|+2}, \\ R_{m}(z) & \text { if } z \in A_{m}, m=1, \ldots,|p|+1,\end{cases}
$$

where $R_{m}$ is the reflection map in the geodesic line $h_{m}$ and $h \circ m$, is an example of hyperbolic finite foldings of degrees $|p|$ and $-|p|$, respectively.

\section{Image sets of foldings of constant curvature surfaces}

An end e of a locally compact space $Y$ is a function which assigns to each compact subset $K \subset Y$ precisely one nonempty component $e(K)$ of the complement $Y-K$, subject to the requirement that $e(K) \supset e(L)$ whenever $K \subset L$. Any set $N \subset Y$ which contains some $e(K)$ is a neighborhood of the end $e$.

Roughly speaking, the number of ends of $Y$ is the number of nonempty connected components of $K \backslash K$ for a sufficiently big compact subset of $Y$. 
For any $f \in \mathscr{I}(S, S)=\mathscr{I}(S)$, we denote by $e_{f}$ the number of ends of the subset $\operatorname{Im}(f)=$ $\{f(x): x \in S\}$.

Proposition 3.1. If $f: X \rightarrow X$ is a proper map and $X$ has one end, then $f(X)$ has one end. Proof. Since $f$ is proper, $f(X)$ cannot be compact and hence has at least one end. Suppose for contradiction that it has more than one end. Then there is a compact set $K$ in $f(X)$ such that $f(X) \backslash K$ has at least two unbounded components $U_{1}$ and $U_{2}$. Since $f$ is proper, $f^{-1}(K)$ is compact. But then $X \backslash f^{-1}(K)$ has at least the two unbounded components $f^{-1}\left(U_{1}\right)$ and $f^{-1}\left(U_{2}\right)$, so $X$ would have at least two ends.

Corollary 3.2. Let $S$ be either the Euclidean plane, the two-sphere $S^{2}$, or the hyperbolic plane $\mathbb{H}^{2}$. If $f \in \mathscr{F}(S)$, then

$$
e_{f}= \begin{cases}0 & \text { if } S=S^{2}, \\ 1 & \text { if } S=\mathbb{R}^{2} \text { or } S=\mathbb{R}^{2} .\end{cases}
$$

Proof. If $f \in \mathscr{F}_{(}\left(S^{2}\right), \operatorname{Im}(f)$ is a bounded subset of $S^{2}$ and so $e_{f}=0$.

Assume now that $f \in \mathscr{F}\left(\mathbb{R}^{2}\right)$. As seen in Proposition 2.4, $f$ is a proper map. The result now follows directly from Corollary 3.2.

Proposition 3.3. Let $S$ be either the euclidian plane, the two-sphere $S^{2}$ or the hyperbolic plane $\mathbb{M}^{2}$. If $f \in \mathscr{I}(S)$ then,

$$
e_{f}= \begin{cases}0 & \text { if } S=S^{2} \\ \text { infinitely many } & \text { if } S=\mathbb{R}^{2} \text { or } S=\mathbb{U}^{2}\end{cases}
$$

Proof. Since $\mathscr{I}\left(S^{2}\right)=\mathscr{F}\left(S^{2}\right)$, by Corollary 3.2, $e_{f}=0$ for any $f \in \mathscr{I}\left(S^{2}\right)$.

Let $S$ be $\mathbb{R}^{2}$ and let $n$ be an arbitrary integer. The isometric foldings whose construction is illustrated in Figure 3.1 are planar isometric foldings with $n$ ends.

This construction can easily be adapted to produce an image with infinitely many ends-each time one retraces to make the vertical columns longer, add one more vertical column.

Assume now $S$ to be the hyperbolic plane and let $n$ be an arbitrary positive integer. Take the $n+2$ hyperbolic points $P_{1}=1$ and $P_{j}=\exp i\left(\pi / 2+\pi / 4+\cdots+\pi / 2^{j-1}\right), j=$ $2, \ldots, n+2$, and denote by $\varrho_{t}$ the reflection on the hyperbolic line $t$.

For $i=1, \ldots, n$, the following hold:

(i) let $r_{i}$ be the hyperbolic line through $P_{i}$ and $P_{i+1}$;

(ii) let $U_{i}, Q_{i}$ be the points determined uniquely by $U_{i}=\varrho_{r_{i}}\left(P_{i+2}\right)=\varrho_{\widehat{P_{i} Q_{i}}}\left(P_{i+1}\right)$;

(iii) let $\left(Q_{i, k}\right)_{k \in \mathbb{N}_{0}}$ be $n$ sequences of hyperbolic points defined by $Q_{i, 0}=P_{i}, Q_{i, 1}=Q_{i}$, $Q_{i, 2}=U_{i}$, and for $k \geq 2, Q_{i, k}=\varrho_{\widehat{P_{i} Q_{i, k-1}}}\left(Q_{i, k-2}\right)$.

The hyperbolic folding, whose set of singularities is

$$
\bigcup_{i=1, \ldots, n ; k \in \mathbb{N}_{0}}\left(\widehat{P_{i} P_{i+1}} \bigcup \widehat{P_{i} Q_{i, k}}\right),
$$


96 Image sets of folding surfaces
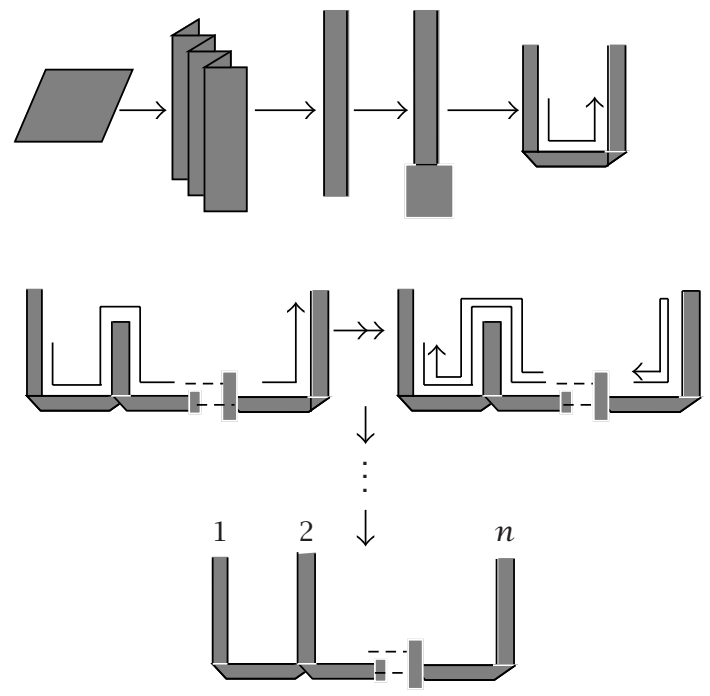

Figure 3.1. Planar folding with $n$ ends.

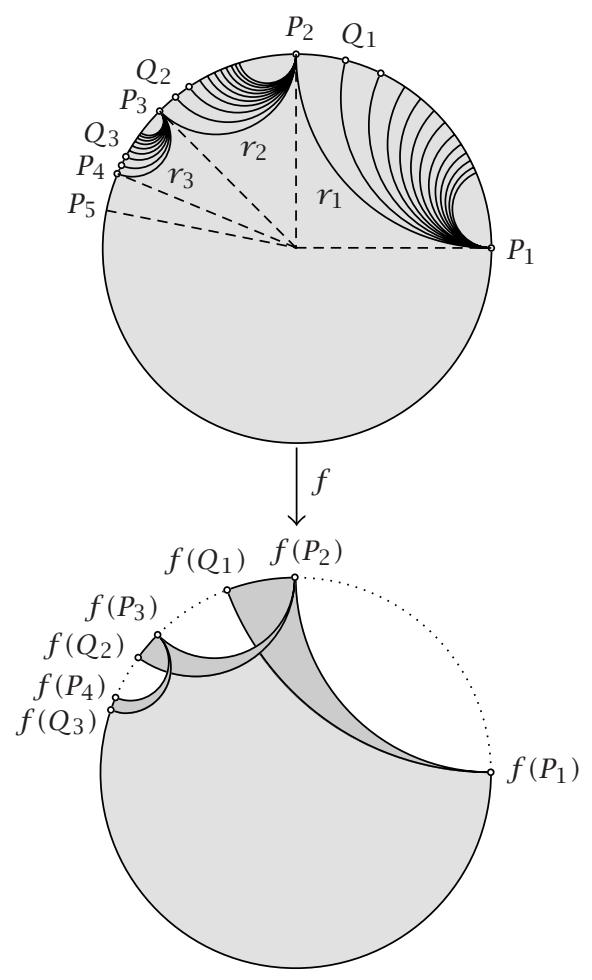

Figure 3.2. Hyperbolic folding with 3 ends. 
is a folding with $n$ ends. In Figure 3.2, the folding process of a hyperbolic folding with 3 ends is illustrated.

As in the previous case, the above construction can easily be adapted to produce a hyperbolic folding with infinitely many ends.

\section{Acknowledgment}

The author is grateful to the referee for his helpful comments and improvements. This work was supported in part by UI\&D "Matemática e Aplicações".

\section{References}

[1] M. W. Hirsch, Differential Topology, Graduate Texts in Math., Springer-Verlag, New York, 1976.

[2] S. A. Robertson, Isometric folding of Riemannian manifolds, Proc. Roy. Soc. Edinburgh Sect. A 79 (1977/78), no. 3-4, 275-284.

Ana M. d'Azevedo Breda: Department of Mathematics, University of Aveiro, 3810-193 Aveiro, Portugal

E-mail address: ambreda@mat.ua.pt 


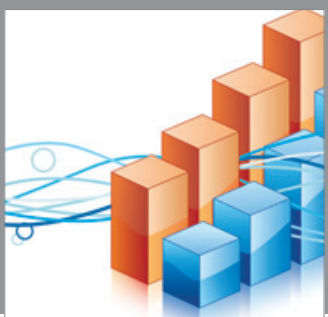

Advances in

Operations Research

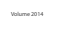

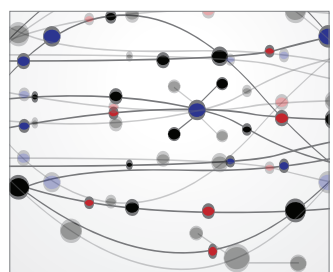

\section{The Scientific} World Journal
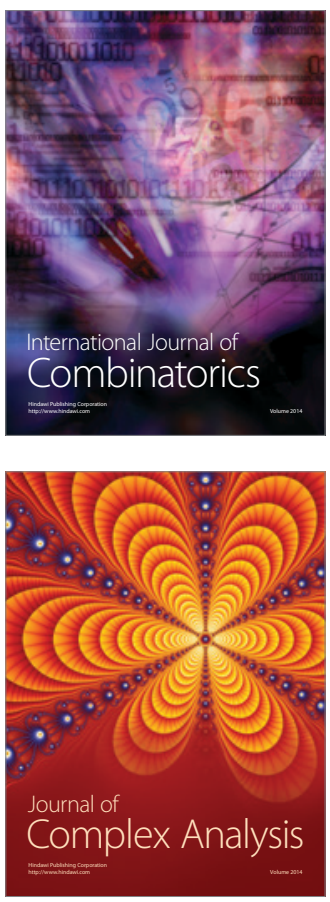

International Journal of

Mathematics and

Mathematical

Sciences
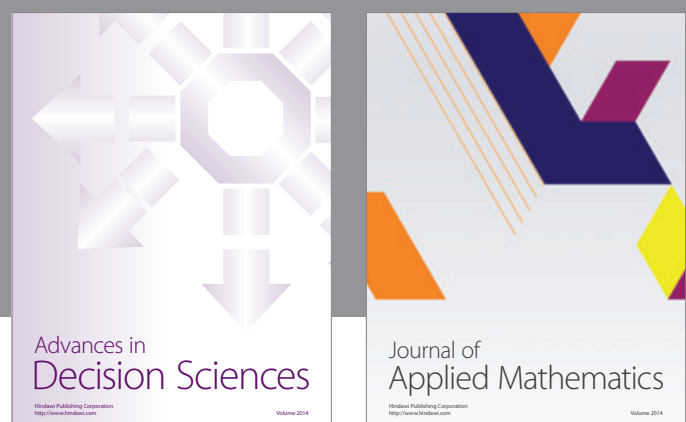

Journal of

Applied Mathematics
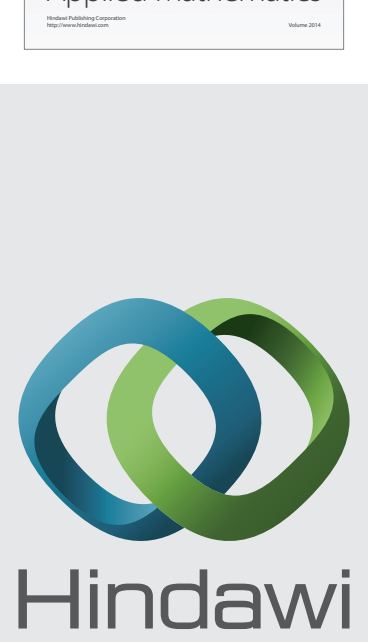

Submit your manuscripts at http://www.hindawi.com
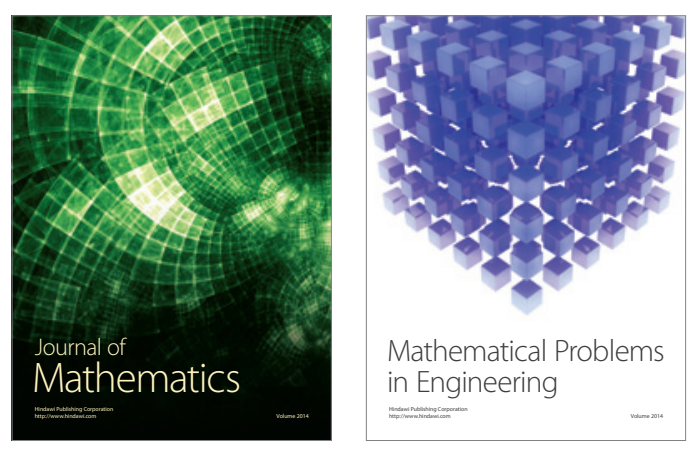

Mathematical Problems in Engineering
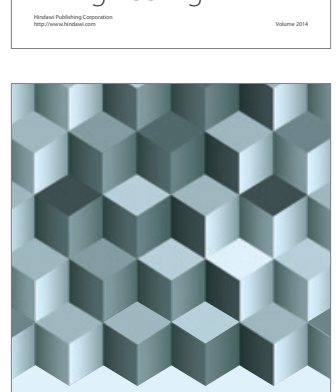

Journal of

Function Spaces
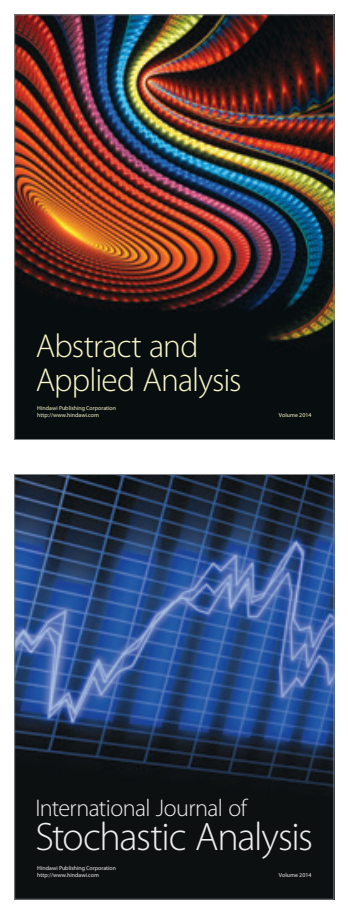

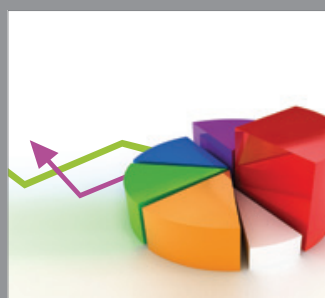

ournal of

Probability and Statistics

Promensencen
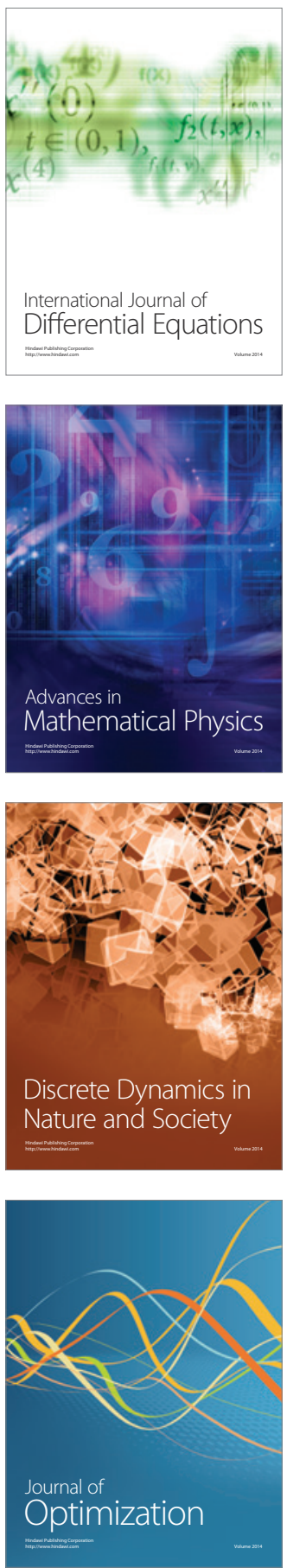